\title{
Model-Free DC Bus Voltage and Energy Management Control for Fuel Cell Hybrid Electric Vehicle
}

\author{
Haoping WANG, Yuzhi LI, Yang TIAN*, Congcong WANG \\ Sino-French International Joint Laboratory of Automatic Control and Signal Processing (LaFCAS), \\ School of Automation, Nanjing University of Science \& Technology, Nanjing 210094, China \\ tianyang@njust.edu.cn (*Corresponding author)
}

\begin{abstract}
In this paper, a model-free DC bus voltage control and energy management strategy are designed for fuel cell hybrid electric vehicle. First, the fuel cell hybrid power system which is composed of fuel cell, battery and supercapacitor is described, and the ultra-local model is constructed based on the input and the output of the system. Then, the model-free continuous fast terminal sliding mode control method is proposed and adopted to design the DC bus voltage controller and sources current controller, and the high-order fast terminal sliding mode observer is designed to estimate the unknown terms of the ultra-local model. Finally, a novel state-based energy management strategy which is composed of cruise state, low power state and charge power state is developed to provide efficient power distribution between different power sources. To verify the performance of the controller, simulations have been done on MATLAB/Simulink, which show that the proposed control strategy ensures good performance and a finite time stability which simultaneously meets all the control objectives.
\end{abstract}

Keywords: Fuel Cell, Hybrid electric vehicle, Model-free control, Energy Management Strategy, Global finite time stability.

\section{Introduction}

In recent years, as environmental pollution and energy shortages have become increasingly serious, fuel cell (FC) vehicles have attracted much attention as new energy vehicles with great development prospects (Larminie, Dicks \& McDonald, 2003; Twidell \& Weir, 2015). However, due to the slow dynamic response and unable to absorb braking energy, the fuel cell stack is usually utilized together with energy storage devices (ESS).

Battery (B) and supercapacitor (SC) are the most widely used ESS in fuel cell hybrid electric vehicle (FCHEV). In most literature, the ESS utilizes only one of them (B or SC) to support the FC. The ESS, which is composed of both SC and battery, is considered here to improve the performance of the hybrid power systems (Das, Tan \& Yatim, 2017; Pany, Singh \& Tripathi, 2016), taking into account the high-energy density of the battery that can provide primary auxiliary power and the highpower density and long lifetime of SC which can quickly respond to load changes.

Meanwhile, the use of this topology which combines the following three sources of energy has the following reason: the $\mathrm{FC}$ will be mainly used to support the average load power of vehicle, the battery will be employed to assist fuel cell to provide peak power or absorb regenerative braking energy, and meanwhile, the supercapacitor will be used to meet the requirements of high-frequency load power changed with rapid speed. Moreover, this hybridization can downsize the fuel cell and battery then reduce the power system cost (Wilberforce et al., 2017; Vural et al., 2014).

In the mentioned multi-source system, because FC, SC, or battery cannot be connected directly to the load, the $\mathrm{DC} / \mathrm{DC}$ power converters are needed between sources and DC bus which also add more degrees of freedom. To ensure the dynamic exchange of energy between the FC stack, load, and ESS modules, the DC/DC converter control can be used (Venkatesh \& Kamalakannan, 2020, Venkatesh \& Kamalakannan, 2016). The control of $\mathrm{FC} / \mathrm{SC} / \mathrm{B}$ hybrid electric vehicle has the following objectives (Mane et al., 2017; Tahri et al., 2018): regulates the DC bus voltage in order to guarantee a stable supply voltage for an electric motor, tracks the reference current signals and guarantees the asymptotic stability of the closedloop system.

Recently, many control methods of FCHEV are put forward. The most widely used is the PI controller for its simplicity and practicality (Aouzellag, Ghedamsi \& Aouzellag, 2015). In (Fadil et al., 2014), a nonlinear control is proposed based on the dynamic model of the system. Depature et al. (2017) propose a stable control for a fuel cell vehicle with SC using a backstepping control technique and demonstrate the global stability of the whole system for real-time implementation. In (Xu et al., 2019a), a terminal sliding mode control with projection operator adaptive law is proposed to get good tracking performance of the DC bus current. Considering the nonlinear behavior 
of power sources and converters, a nonlinear controller using the Lyapunov stability approach is developed (Benmouna et al., 2020). The numerical simulations prove that the developed controller meets its performances.

Recently, the model-free control (MPC) method (Mustafa, Wang \& Tian, 2019) has attracted the attention of some researchers as it reduces the dependence on model information, when compared with the model-based control method. The basic idea of MFC control is to divide the general system model into the ultra-local model with the input and output of the system (Fliess \& Join, 2013; Barkat et al., 2018). In (Zhao et al., 2019), aiming at the problem of permanent magnet (PM) demagnetization, a model-free controller is designed based on an ultra-local model, and the sliding mode observer is used to estimate the unknown states. However, the fuel cell hybrid power system was managed without using the MFC control technique. Furthermore, the MFC control was designed in a global approach with a mathematical state representation.

In this paper, a model-free continuous fast terminal sliding mode control (MFCFTSMC) method is proposed, which combines model-free control (Fliess \& Join, 2013) with continuous fast terminal sliding mode control (Wang et al., 2018). It uses the high-order fast terminal sliding mode (HOFTSM) observer (Xu et al., 2019b) to ensure reliable operation of the FCHEV power system without being affected by model uncertainty and external disturbances. The ultra-local model based on the input and output of permanent magnet synchronous motor (PMSM) is established, to reduce the dependence of the controller on the precise power system model. The objective of controller design is to obtain stable DC bus voltage and a good current tracking for the two energy sources. Moreover, a state-based energy management strategy (EMS) (Song et al., 2018; Marzougui et al., 2017) which is composed of cruise state, low power state and charge power state is developed to provide efficient power distribution between different power sources. Compared with the previous work, the proposed method can improve the working efficiency of the fuel cell and reduce the fuel consumption of the energy system, thereby improving the performance of the vehicle.
The FCHEV as well as the model-free controller and energy management are modeled in MATLAB/Simulink environment using the Simscape Power Systems (SPS) toolbox. The rest of the paper is organized as follows. Section 2 describes the ultra-local modeling of fuel cell hybrid systems. Section 3 describes the controller design and analysis of the fuel cell hybrid system. Section 4 gives the procedure to design the statebased energy management strategy. Simulation results are presented in Section 5. Finally, the conclusions of the study are summarized in Section 6 .

\section{Ultra-local Model for Fuel Cell Hybrid System}

This section aims to develop a large-signal model of the power circuit of the fuel cell hybrid power system taking into account its nonlinearities. Based on the input and output measurement results of the system, the ultra-local model will be constructed for being used later in the control design.

Figure 1 shows the schematic of the power stage of the proposed FCHEV which consists in a boost and a buck-boost converter for fuel cell, battery and supercapacitor, respectively. The function of the fuel cell is to supply mean power to the load, whereas the storage device is used as a power source: it supplies transient power demand, and peak loads required during acceleration and deceleration.

The mathematical model of boost converter can be expressed by (Armghan et al., 2018):

$$
\begin{aligned}
& \frac{d i_{f c}}{d t}=\frac{v_{f c}}{L_{f c}}-\left(1-u_{1}\right) \frac{v_{o u t}}{L_{f c}}-\frac{R_{f c}}{L_{f c}} i_{f c}+\theta_{1} \\
& i_{f c_{-} \text {out }}=u_{1} i_{f c}
\end{aligned}
$$

where $\theta_{1}$ represents the model inaccuracies or external disturbance, $i_{f c}$ is the current passing through inductor $L_{f c}, R_{f c}$ is the boost converter resistance, $v_{f_{c}}$ is the fuel cell voltage, $u_{1}$ is the control input for switch $\mathrm{S}_{1}, v_{\text {out }}$ is the output voltage of DC bus, and $i_{f c_{\text {_out }}}$ is the output current of the boost converter. The converter that connects the battery to DC bus voltage is a two-switch bidirectional buck-boost converter. Buck-boost converter works as a boost converter in discharging mode and as a buck converter in charging mode. $\mathrm{K}$ is defined as: 


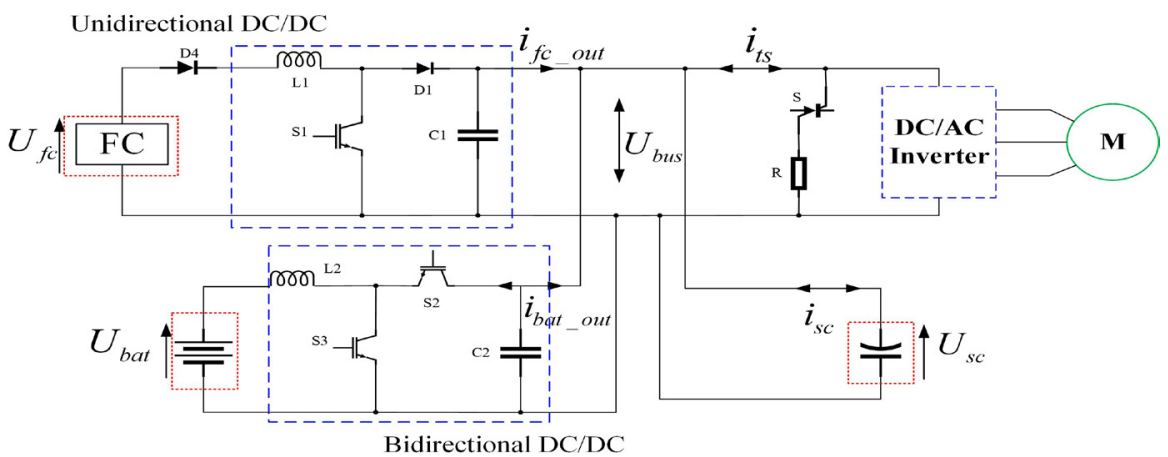

Figure 1. Power stage schematic of the proposed FCHEV

$K= \begin{cases}1, & \text { if } \mathrm{i}_{\text {batref }}>0 \text { (Boost mode) } \\ 0, & \text { if } \mathrm{i}_{\text {batref }}<0 \text { (Buck mode) }\end{cases}$

The virtual control is defined as follows:

$u_{23}=\left[K\left(1-u_{2}\right)+(1-K) u_{3}\right]$

where $u_{2}$ is the control input for switch $\mathrm{S}_{2}$ and $u_{3}$ is the control input for switch $\mathrm{S}_{3}$.

Hence, the battery converter model can be defined by the following pair of differential equations (Hilairet et al., 2013):

$$
\begin{aligned}
& \frac{d i_{b a t}}{d t}=\frac{v_{b a t}}{L_{b a t}}-u_{23} \frac{v_{o u t}}{L_{b a t}}-\frac{R_{b a t}}{L_{b a t}} i_{b a t}+\theta_{3} \\
& \frac{d V_{b u s}}{d t}=u_{23} \frac{i_{b a t}}{C_{d c}}+\left(1-u_{1}\right) \frac{i_{f c}}{C_{d c}}-\frac{i_{t s}-i_{s c}}{C_{d c}}+\theta_{3} \\
& i_{\text {bat_out }}=u_{23} i_{b a t}
\end{aligned}
$$

where $\theta_{2}$ and $\theta_{3}$ represent the model inaccuracies or external disturbance, $i_{b a t}$ is the current passing through inductor $L_{b a t}, i_{s c}$ is the supercapacitor current, $i_{t s}$ is the demand current, $i_{b a t \text { out }}$ is the output current of the buck-boost converter, $R_{b a t}$ is the buck-boost converter resistance, $v_{b a t}$ is battery voltage, $v_{\text {bus }}$ is the DC bus voltage, $u_{23}$ is the virtual control input for switches $\mathrm{S}_{2}$ and $\mathrm{S}_{3}$, and $C_{d c}$ is the capacitor of DC bus.

By combining (6), (7) and (4), the following bilinear switched model of the global system is obtained:

$$
\left\{\begin{array}{l}
\frac{d i_{f c}}{d t}=\frac{v_{f c}}{L_{f c}}-\left(1-u_{1}\right) \frac{v_{o u t}}{L_{f c}}-\frac{R_{f c}}{L_{f c}} i_{f c}+\theta_{1} \\
\frac{d i_{b a t}}{d t}=\frac{v_{b a t}}{L_{b a t}}-u_{23} \frac{v_{o u t}}{L_{b a t}}-\frac{R_{b a t}}{L_{b a t}} i_{b a t}+\theta_{2} \\
\frac{d V_{b u s}}{d t}=u_{23} \frac{i_{b a t}}{C_{d c}}+\left(1-u_{1}\right) \frac{i_{f c}}{C_{d c}}-\frac{i_{t s}-i_{s c}}{C_{d c}}+\theta_{3}
\end{array}\right.
$$

It is used in order to reduce the dependence of the bus voltage controller and current controller on the fuel cell hybrid system model and the impact of un-modeled dynamics or parametric perturbations on the controller. The ultra-local model of the bus voltage loop and source current loop for fuel cell hybrid system can be designed as follows:

$$
\left\{\begin{array}{l}
F_{b u s} \frac{d i_{f c}}{d t}=\alpha_{f c} u_{1}+F_{f c} \\
\frac{d i_{b a t}}{d t}=\alpha_{b a t} u_{23}+F_{b a t} \\
\frac{d V_{b u s}}{d t}=\alpha_{b u s} i_{b a t}+F_{b u s}
\end{array}\right.
$$

where $\alpha_{b u s}$ is the designed voltage parameter, $\alpha_{f c}$ and $\alpha_{b a t}$ are the designed current parameter; $F_{b u s}$, $F_{f c}, F_{b a t}$ have known parts of the mathematical model of fuel cell hybrid system in normal condition and un-modeled dynamics or parametric perturbations, and they are all bounded quantities.

According to the ultra-local model, the equation can be rearranged as follows:

$\dot{x}=\alpha u+F$

with $\quad \boldsymbol{x}=\left[\begin{array}{lll}i_{f c} & i_{b a t} & V_{b u s}\end{array}\right]^{T}, \quad \boldsymbol{\alpha}=\operatorname{diag}\left(\alpha_{f c}, \alpha_{b a t}, \alpha_{b u s}\right)$, $\boldsymbol{u}=\left[\begin{array}{lll}u_{1} & u_{23} & i_{b a t}\end{array}\right]^{T}$ and $F=\left[\begin{array}{lll}F_{f c} & F_{b a t} & F_{b u s}\end{array}\right]^{T}$.

The ultra-local model of fuel cell hybrid power system is constructed. The high-order fast terminal sliding mode observer (HOFTSMO) is used to estimate the variable $\hat{F}$ and send the feedback to the MFCFTSMC controller. The MFCFTSMC method can be used to design the energy sources current controllers and DC bus voltage controller without depending on the specific model of the fuel cell hybrid power system. 


\section{MFCFTSMC Method with HOFTSMO}

To obtain higher steady-state tracking accuracy, reduce the dependence of the design of the controller on the precise fuel cell hybrid system model, the MFCFTSMC method is proposed in this section as illustrated in Figure 2.

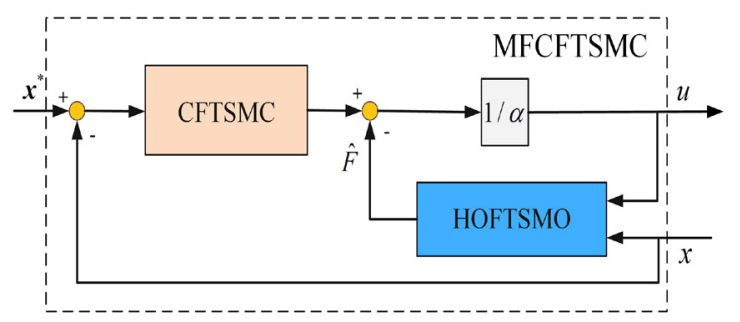

Figure 2. The control structure of MFCFTSMC

Its schematic diagram includes MFCFTSMC controller and HOFTSMO. The HOFTSMO is used to estimate the variances of the ultra-local model and send the feedback of it to the controller. The controller is used to control DC bus voltage and source current. The MFAFTSMC method can be used to design the battery, fuel cell current controllers and bus voltage controller for fuel cell hybrid control system.

\subsection{MFCFTSMC Controller Design}

The state error of the controller is designed as follows:

$e_{1}=x^{*}-x$

where $\boldsymbol{e}_{1}=\left[\begin{array}{lll}e_{f c} & e_{b a t} & e_{b u s}\end{array}\right]^{T}$, and $e_{f c}=i_{f c}^{*}-i_{f c}$, $e_{\text {bat }}=i_{\text {bat }}^{*}-i_{\text {bat }}, e_{\text {bus }}=V_{\text {bus }}^{*}-V_{\text {bus }} \cdot \boldsymbol{x}^{*}=\left[\begin{array}{lll}i_{f c}^{*} & i_{\text {bat }}^{*} & V_{\text {bus }}^{*}\end{array}\right]^{T}$, and $i_{f c}^{*}, i_{b a t}^{*}$ are the given value of fuel cell and battery current, $V_{b u s}^{*}$ is the given value of the DC bus voltage.

According to equations (10) and (11), the statespace equation is as follows:

$\dot{\boldsymbol{e}}_{1}=\dot{\boldsymbol{x}}^{*}-\boldsymbol{\alpha u}-\boldsymbol{F}$

To improve the convergence speed of the state, a continuous fast terminal sliding mode manifold is designed as follow:

$\boldsymbol{s}_{1}=\dot{\boldsymbol{e}}_{1}+{ }_{1 / 4}\left|\dot{\boldsymbol{e}}_{1}\right|^{\sigma_{1}} \operatorname{sign}\left(\dot{\boldsymbol{e}}_{1}\right)+\cdot\left|\boldsymbol{e}_{1}\right|^{\sigma_{2}} \operatorname{sign}\left(\boldsymbol{e}_{1}\right)$

where $\boldsymbol{\mu}=\operatorname{diag}\left(\mu_{1}, \mu_{2}, \mu_{3}\right), \boldsymbol{\eta}=\operatorname{diag}\left(\eta_{1}, \eta_{2}, \eta_{3}\right)$, $\mu_{1}>0, \quad \mu_{2}>0, \quad \mu_{3}>0, \eta_{1}>0, \eta_{2}>0$, $\eta_{3}>0$ are the designed parameter; $0<\sigma_{1}<2$, $\sigma_{1}<\sigma_{2}$.
Supposition: Define the observer error $\tilde{\boldsymbol{F}}=\hat{\boldsymbol{F}}-\boldsymbol{F}$. Take the derivative of $\tilde{\boldsymbol{F}}$, which is bounded, and suppose that there exists a constant $\xi_{a}$ such that $\|\dot{\tilde{\boldsymbol{F}}}\| \leq \boldsymbol{\xi}_{\boldsymbol{a}}$ is true for all $t \geq 0$.

Theorem 1: For (11), the state error can converge to zero in finite time, if the CFTSM manifold is chosen as equation (13) and the control law (14) is designed as follows:

$\boldsymbol{u}=\boldsymbol{\alpha}^{-1}\left(\chi_{e q}+\chi_{b}\right)$

$\chi_{e q}=\dot{\boldsymbol{x}}^{*}+\boldsymbol{\mu}\left|\dot{\boldsymbol{e}}_{1}\right|^{\sigma_{1}} \operatorname{sign}\left(\dot{\boldsymbol{e}}_{1}\right)+\boldsymbol{\eta}\left|\boldsymbol{e}_{1}\right|^{\sigma_{2}} \operatorname{sign}\left(\boldsymbol{e}_{1}\right)-\hat{\boldsymbol{F}}$

$\chi_{b}=\int_{0}^{t} \boldsymbol{k} \boldsymbol{s}_{1}+\boldsymbol{h}\left\|\boldsymbol{s}_{1}\right\|^{\sigma_{3}} \operatorname{sign}\left(\boldsymbol{s}_{1}\right) d t$

where $\hat{\boldsymbol{F}}$ is the estimated value of $F$, $\boldsymbol{k}=\operatorname{diag}\left(k_{1}, k_{2}, k_{3}\right), \boldsymbol{h}=\operatorname{diag}\left(h_{1}, h_{2}, h_{3}\right), k_{1}>0$ , $k_{2}>0, k_{3}>0, h_{1}>0, h_{2}>0, h_{3}>0$ are the designed parameters.

Proof: By substituting (13) into (12), the following equation is obtained:

$$
\begin{aligned}
\boldsymbol{s}_{1} & =\dot{\boldsymbol{e}}_{1}+\boldsymbol{\mu}\left|\dot{\boldsymbol{e}}_{1}\right|^{\sigma_{1}} \operatorname{sign}\left(\dot{\boldsymbol{e}}_{1}\right)+\boldsymbol{\eta}\left|\boldsymbol{e}_{1}\right|^{\sigma_{2}} \operatorname{sign}\left(\boldsymbol{e}_{1}\right) \\
& =\dot{\boldsymbol{x}}^{*}-\boldsymbol{\alpha u}-\boldsymbol{F}+\boldsymbol{\mu}\left|\dot{\boldsymbol{e}}_{1}\right|^{\sigma_{1}} \operatorname{sign}\left(\dot{\boldsymbol{e}}_{1}\right)+\boldsymbol{\eta}\left|\boldsymbol{e}_{1}\right|^{\sigma_{2}} \operatorname{sign}\left(\boldsymbol{e}_{1}\right)
\end{aligned}
$$

According to equation (14), the following equation is obtained:

$$
\begin{aligned}
s_{1} & \dot{x^{*}}-\alpha\left[\alpha^{-1}\left(\chi_{e q}+\chi_{b}\right)\right]-\boldsymbol{F}+\mu\left|\dot{\boldsymbol{e}}_{1}\right|^{\sigma_{1}} \operatorname{sign}\left(\dot{\boldsymbol{e}}_{1}\right) \\
& +\boldsymbol{\eta}\left|\boldsymbol{e}_{1}\right|^{\sigma_{2}} \operatorname{sign}\left(\boldsymbol{e}_{1}\right)
\end{aligned}
$$

By simplifying the above equation, one has

$s_{1}=-\chi_{b}+\hat{\boldsymbol{F}}-\boldsymbol{F}$

The following Lyapunov function is selected as:

$V_{1}=\frac{1}{2} s_{1}^{T} s_{1}$

By differentiating $V_{1}$ with respect to time, substituting (20) into it and defining $\tilde{\boldsymbol{F}}=\hat{\boldsymbol{F}}-\boldsymbol{F}$, the following equation is obtained:

$$
\begin{aligned}
\dot{\boldsymbol{V}}_{1} & =\frac{1}{2} \boldsymbol{s}_{1}^{T} \boldsymbol{s}_{1}=\boldsymbol{s}_{1}^{T}\left(-\boldsymbol{k} \boldsymbol{s}_{1}-\boldsymbol{h}\left|\boldsymbol{s}_{1}\right|^{\sigma_{3}} \operatorname{sign}\left(\boldsymbol{s}_{1}\right)+\dot{\tilde{\boldsymbol{F}}}\right) \\
& \leq-\left(\boldsymbol{h}-\frac{\dot{\tilde{\boldsymbol{F}}}}{\left\|\boldsymbol{s}_{1}\right\|^{\sigma_{3}}}\right)\left\|\boldsymbol{s}_{1}\right\|^{\sigma_{3}+1}-\boldsymbol{k}\left\|\boldsymbol{s}_{1}\right\|^{2} \\
& =-2 \beta_{1} V_{1}-2^{\frac{\sigma_{3}+1}{2}} \beta_{2} V_{1}^{\frac{\sigma_{3}+1}{2}}
\end{aligned}
$$

According to (21), it can be confirmed that $\boldsymbol{h}>\boldsymbol{\xi}_{\boldsymbol{a}}$, and the tracking error would reach the sliding surface when $\boldsymbol{s}_{1}=0$. Equation (17) can be 
designed as the MFCFTSMC which would converge in finite time, and the trajectory of the state would stay in the sliding surface. Therefore, the states would be converged to zero in finite time, hence the Lyapunov function can be satisfied. This completes the proof.

\subsection{HOFTSMO Observer}

In equation (21), $\boldsymbol{F}$ is the unknown term and the HOFTSMO observer is designed to obtain the estimated value. Regarding $\boldsymbol{F}$ as the system extended state, the fuel cell hybrid system can be expressed as:

$\left\{\begin{array}{l}\dot{x}=\alpha u+F \\ \dot{F}=a\end{array}\right.$

where $\boldsymbol{a}$ is the derivative of the unknown term $\boldsymbol{F}$.

The sliding mode observer can be designed as follows:

$\left\{\begin{array}{l}\dot{\hat{\boldsymbol{x}}}=\boldsymbol{\alpha} \boldsymbol{u}+\hat{\boldsymbol{F}}+\boldsymbol{P} \\ \dot{\hat{\boldsymbol{F}}}=\boldsymbol{Q}\end{array}\right.$

where $\wedge$ presents the estimated value, $\boldsymbol{P}$ is the designed control law; and $\boldsymbol{Q}$ is the designed equivalent for the derivative of the unknown term $F$.

By subtracting (23) from (22), we can obtain the system error state equation as follows:

$\left\{\begin{array}{l}\dot{\boldsymbol{e}}_{x}=\boldsymbol{e}_{F}-\boldsymbol{P} \\ \dot{\boldsymbol{e}}_{F}=\boldsymbol{a}-\boldsymbol{Q}\end{array}\right.$

where $\boldsymbol{e}_{x}=\boldsymbol{x}-\hat{\boldsymbol{x}}$ is the state estimated error, and $\boldsymbol{e}_{F}=\boldsymbol{F}-\hat{\boldsymbol{F}}$ is the unknown term estimated error. A FTSM surface is designed to achieve finite time convergence and better tracking accuracy.

$\boldsymbol{S}_{2}=\dot{\boldsymbol{e}}_{x}+\lambda \boldsymbol{e}_{x}+\boldsymbol{\gamma}\left\|\boldsymbol{e}_{x}\right\|^{\sigma_{4}} \operatorname{sign}\left(\boldsymbol{e}_{x}\right)$

where $\lambda=\operatorname{diag}\left(\lambda_{1}, \lambda_{2}, \lambda_{3}\right), \boldsymbol{\gamma}=\operatorname{diag}\left(\gamma_{1}, \gamma_{2}, \gamma_{3}\right)$, $\lambda_{1}>0, \lambda_{2}>0, \lambda_{3}>0, \gamma_{1}>0, \gamma_{2}>0, \gamma_{3}>0$, and $0<\sigma_{4}<1$ are the designed parameters.

Theorem 2: For the error system (24), the FTSM surface (25) will be reached, and the states $\boldsymbol{e}_{x}$ and $\dot{\boldsymbol{e}}_{x}$ can converge to 0 along the FTSM surface within finite time, if the HOSM control law is designed as follows:
$\boldsymbol{P}=\lambda \boldsymbol{e}_{x}+\boldsymbol{\gamma}\left\|\boldsymbol{e}_{x}\right\|^{\sigma_{4}} \operatorname{sign}\left(\boldsymbol{e}_{x}\right)+\boldsymbol{P}_{n}$

$\dot{\boldsymbol{P}}_{n}+\omega_{f} \boldsymbol{P}_{n}=\boldsymbol{m} \operatorname{sign}\left(\boldsymbol{s}_{2}\right)$

where $\boldsymbol{P}_{n}(0)=0, \boldsymbol{m}=\operatorname{diag}\left(m_{1}, m_{2}, m_{3}\right)$, and $\omega_{f}$ are the designed parameters of the control law. The equivalent for the derivative of the unknown term is designed as follow:

$\boldsymbol{Q}=\boldsymbol{n} \operatorname{sign}\left(\boldsymbol{s}_{2}\right)$

where $\boldsymbol{n}=\operatorname{diag}\left(n_{1}, n_{2}, n_{3}\right)$ is the designed parameter.

Proof: Consider a Lyapunov function:

$\boldsymbol{V}_{2}=\frac{1}{2} \boldsymbol{s}_{2}^{T} \boldsymbol{s}_{2}$

The sliding mode surface $\boldsymbol{s}_{2}$ can be rewritten by substituting the first equation of into as follows:

$$
\begin{aligned}
\boldsymbol{S}_{2} & =\boldsymbol{e}_{F}-\boldsymbol{P}+\lambda \boldsymbol{e}_{x}+\boldsymbol{\gamma}\left\|\boldsymbol{e}_{x}\right\|^{\sigma_{4}} \operatorname{sign}\left(\boldsymbol{e}_{x}\right) \\
& =\boldsymbol{e}_{F}-\lambda \boldsymbol{e}_{x}-\boldsymbol{\gamma}\left\|\boldsymbol{e}_{x}\right\|\left\|^{\sigma_{4}} \operatorname{sigh}\left(\boldsymbol{e}_{x}\right)-\boldsymbol{P}_{n}+\lambda \boldsymbol{e}_{x}+\boldsymbol{\gamma}\right\| \boldsymbol{e}_{x} \|^{\sigma_{4}} \operatorname{sign}\left(\boldsymbol{e}_{x}\right) \\
& =\boldsymbol{e}_{F}-\boldsymbol{P}_{n}
\end{aligned}
$$

Then, the derivative of $\boldsymbol{s}_{2}$ with respect to time $t$ can be expressed as follows:

$$
\begin{aligned}
& \dot{\boldsymbol{s}}_{2}=\dot{\boldsymbol{e}}_{F}-\dot{\boldsymbol{P}}_{n} \\
& =\boldsymbol{a}-\boldsymbol{Q}-\boldsymbol{m} \operatorname{sign}\left(\boldsymbol{s}_{2}\right)+\omega_{f} \boldsymbol{P}_{n} \\
& =-(\boldsymbol{m}+\boldsymbol{n}) \operatorname{sign}\left(\boldsymbol{s}_{2}\right)+\omega_{f} \boldsymbol{P}_{n}+\boldsymbol{a}
\end{aligned}
$$

The derivative of $\boldsymbol{V}_{2}$ with respect to time $t$ can be given by the following:

$\dot{\boldsymbol{V}}_{2}=\boldsymbol{s}_{2}^{T}\left(-(\boldsymbol{m}+\boldsymbol{n}) \operatorname{sign}\left(\boldsymbol{s}_{2}\right)+\omega_{f} \boldsymbol{P}_{n}+\boldsymbol{a}\right)$

$=-(\boldsymbol{m}+\boldsymbol{n})\left\|\boldsymbol{s}_{2}\right\|+\omega_{f} \boldsymbol{P}_{n} \boldsymbol{s}_{2}^{T}+\boldsymbol{a} \boldsymbol{s}_{2}^{T}$

$=\left(-\boldsymbol{m}\left\|\boldsymbol{s}_{2}\right\|+\omega_{f} \boldsymbol{P}_{n} \boldsymbol{s}_{2}^{T}\right)+\left(-\boldsymbol{n}\left\|\boldsymbol{s}_{2}\right\|+\boldsymbol{a} \boldsymbol{s}_{2}^{T}\right)$

By selecting $\boldsymbol{m}>\left\|\omega_{f} \boldsymbol{P}_{n}\right\|, \boldsymbol{n}>\|\boldsymbol{a}\|+\varepsilon$, and $\varepsilon$ is a positive constant. For $\boldsymbol{s}_{2} \neq 0$ we obtain the following:

$\dot{\boldsymbol{V}}_{2}=\boldsymbol{s}_{2}^{T} \dot{\boldsymbol{s}}_{2}<-\varepsilon\left\|\boldsymbol{s}_{2}\right\|<0$

Therefore, based on the Lyapunov stability theorem, the system dynamics can converge to the FTSM surface (25) within finite time, and stay on it thereafter and then the finite time convergence of the states $\boldsymbol{e}_{x}$ and $\dot{\boldsymbol{e}}_{x}$ to 0 is ensured by applying the FTSM surface. 


\section{Energy Management Strategy}

A state-based energy management strategy (EMS) which is composed of cruise state, low power state and charge power state is developed in this section. The task of the state-based EMS is to generate fuel cell reference current that completes the power distribution.

The basic idea of the state-based energy management strategy is that the operation of FCHEV is divided into three states according to the values of the load power, and each state adopts a different EMS. By considering the three operating states of FCHEV, the operating characteristics of hybrid power system are different in each state. As shown in Figure 3, based on the power requirements of vehicle driving cycle, three main power states have been divided: cruise management (CM), low power management (LPM) and charge power management (CPM). Fuzzy logic parameter turning based single PI during CM, chargedepleting charge-sustaining (CDCS) (Bassam et al., 2017) during LPM and improved PI (iPI) EMS (Xu et al., 2013) during CPM are used to compose the state-based EMS.

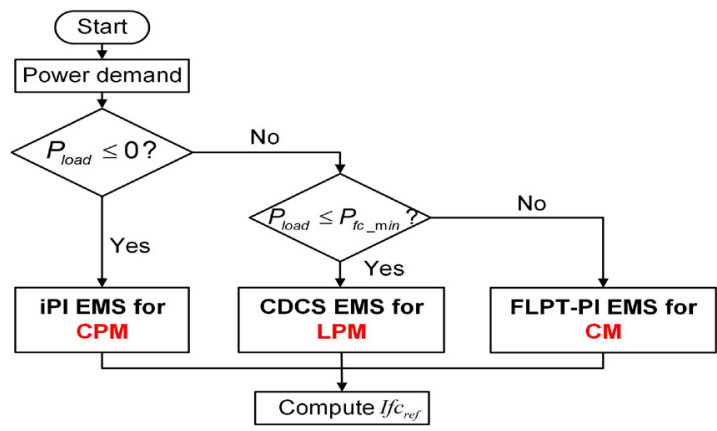

Figure 3. Flow chart of proposed multi-state EMS

\subsection{FLPT-PI EMS Based Cruise State}

The time when the vehicle is in CM accounts for a higher proportion of the entire driving cycle, but the normal PI-based EMS which has the constants $K_{p}, K_{i}$ cannot achieve efficient performance in such a long load power range. To further dividing the cruise state, the fuzzy logic parameter turning (FLPT) is used to separate the wide power range. Compared with the normal PI controller with constants $K_{p}, K_{i}$, the FLPT can dynamically adjust the key-parameters $K_{p}, K_{i}$ which are directly related to the performance of PI control energy management strategy. The schematic diagram of the FLPT-PI strategy is shown in Figure 4.

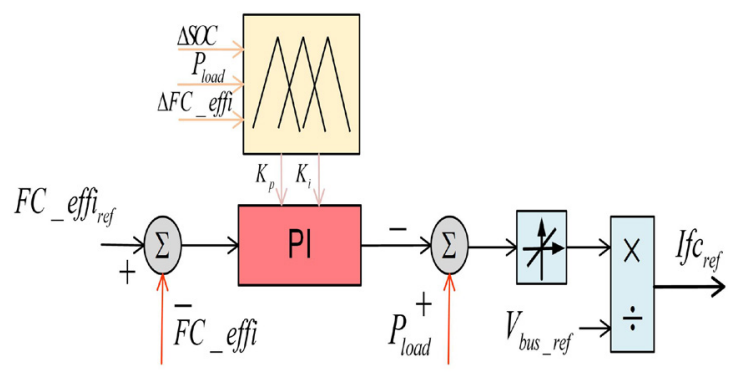

Figure 4. FLPT based single PI control energy management strategy scheme

The input variables of FLPT are chosen as: load power, the deviation of the SOC of the battery and the efficiency of the FC which are defined as:

$\triangle S O C=S O C_{r e f}-S O C$

$\Delta F C_{e f f i}=F C_{e f f i-r e f}-F C_{e f f i}$

Here the output of fuzzy logic controller is the adjustment factor which multiplies the amplification factor and adds it to $K_{p}$ and $K_{i}$. Because $K_{i}$ changes in the same manner as $K_{p}, K_{p}$ is multiplied by the ratio factor to form $K_{i}$. The membership functions (MFs) of the input and output variables are depicted in Figure 5.

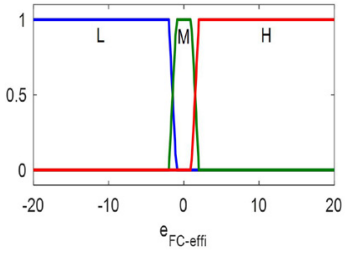

(a)

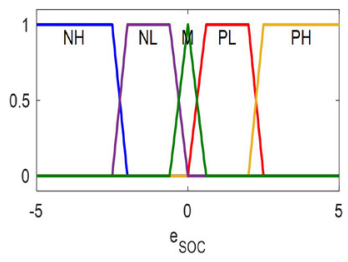

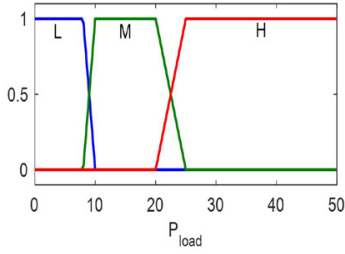

(b)

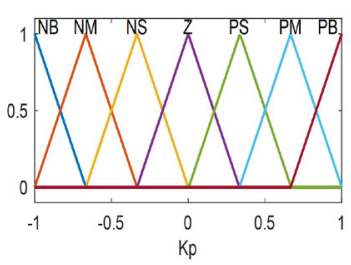

Figure 5. Membership functions of input and output variables: (a) input variable $e_{f c}-e_{f f i}$; (b) input variable $P_{\text {load }} ;$ (c) input variable $e_{\text {soc }} ;$ (d) output variable $K_{p}$

Then, three base rules are defined for those threeload power situations, respectively, as shown in Table 1. 
Table 1. Base rules: (a) low load power state base rule; (b) middle load power state base rule; (c) high load power state base rule

\begin{tabular}{|c|c|c|c|c|c|c|c|c|c|c|c|}
\hline \multicolumn{6}{|c|}{ (a) } & \multicolumn{6}{|c|}{ (b) } \\
\hline$P_{\text {batar }} e_{e_{0 x}}$ & $\mathrm{PH}$ & PL & M & $\mathrm{NL}$ & $\mathrm{NH}$ & 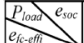 & $\mathrm{PH}$ & PL & $\mathrm{M}$ & $\mathrm{NL}$ & $\mathrm{NH}$ \\
\hline $\mathrm{L}$ & PB & PM & PM & PS & z & $\mathrm{L}$ & PM & PS & PS & $\mathrm{z}$ & NS \\
\hline M & PM & PS & PS & z & NS & $\mathrm{M}$ & PS & z & Z & NS & NM \\
\hline $\mathrm{H}$ & PS & $z$ & NS & NS & $\mathrm{NM}$ & $\mathrm{H}$ & z & NS & NS & NM & $\mathrm{NH}$ \\
\hline
\end{tabular}

\begin{tabular}{|c|c|c|c|c|c|}
\hline \multicolumn{6}{|l|}{$B$} \\
\hline 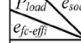 & PH & PL & M & $\mathrm{NL}$ & $\mathrm{NH}$ \\
\hline L & PS & $\mathrm{z}$ & Z & NS & NM \\
\hline M & $\mathrm{z}$ & $\mathrm{z}$ & NS & NM & NM \\
\hline $\mathrm{H}$ & NS & NS & NM & $\mathrm{NM}$ & NB \\
\hline
\end{tabular}

In this subsection, the logic rules are determined by different purposes. In low load power situation, because the range of load power fluctuations is very close to the FC high efficiency area, the main purpose is to pursue the efficiency of FC. Thus, the value of $K_{p}$ increases which makes it more sensitive to changes in FC efficiency. However, in high-load power situation, if the fuel cell efficiency is still excessively expected, this may cause harmful effects of excessive battery discharge. For this reason, the value of $K_{p}$ is designed to be reduced appropriately. In middleload power situation, keeping $K_{p}$ in a balanced value can achieve a compromise effect.

\subsection{CDCS EMS Based Low Power State}

Through the analysis in above subsection, the CDCS strategy, one of the widely used strategies, is applied in order to achieve the load power supply. While the fuel cell stark starts or works at its minimum power, the load power is supplied by the battery until the SOC of the battery decreases to the lower limit.

The schematic diagram of CDCS strategy is shown in Figure 6.

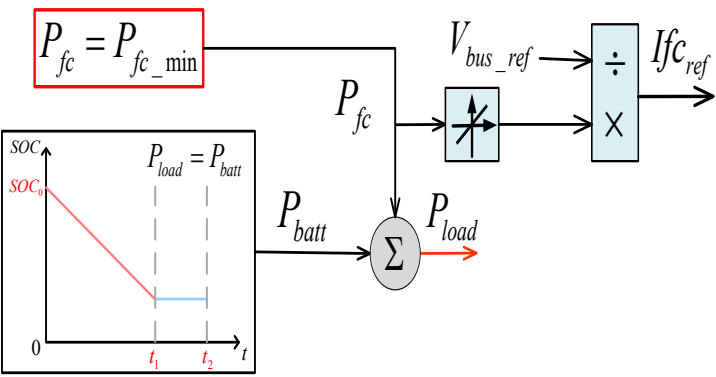

Figure 6. Charge-depleting charge-sustaining strategy scheme

\subsection{Improved PI EMS Based Charge Power State}

Here the improved PI control energy management strategy scheme has been adopted as shown in Figure 7. Compared with the efficiency of the aforementioned single PI control FC module, the SOC of the battery is maintained near its reference value by increasing the control of the SOC of the battery. Therefore, the limitation of restricting the SOC of the battery around its reference value can be achieved.

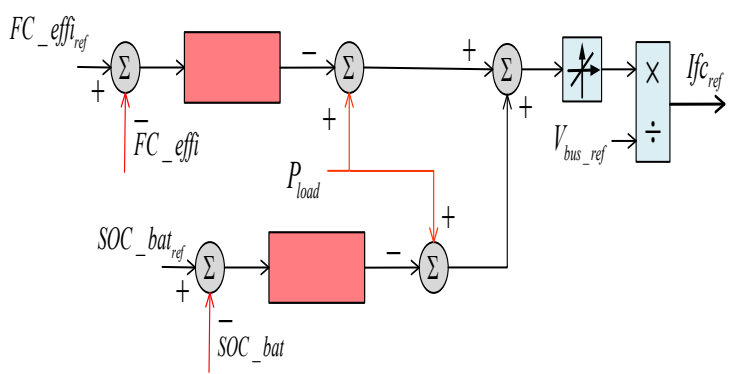

Figure 7. Improved PI control energy management strategy scheme

\section{Results and Discussion}

The effectiveness of the proposed control strategy for FCHEV is verified in the MATLAB Simulink environment. The parameters of the controller are summarized in Table 2.

Table 2. The parameters about controller

\begin{tabular}{|c|c|c|}
\hline & Parameters & Values \\
\hline \multirow{4}{*}{ Gains of } & $\mu_{1}, \mu_{2}, \mu_{3}$ & $1 \mathrm{e}-6,1 \mathrm{e}-7,1 \mathrm{e}-8$ \\
\cline { 2 - 3 } & $\eta_{1}, \eta_{2}, \eta_{3}$ & $1.5,0.015,0.125$ \\
\cline { 2 - 3 } controller & $k_{1}, k_{2}, k_{3}$ & $1,1,0.12$ \\
\cline { 2 - 3 } & $h_{1}, h_{2}, h_{3}$ & $1,1,0.008$ \\
\cline { 2 - 3 } Gains of & $\sigma_{1}, \sigma_{2}, \sigma_{3}$ & $5 / 3,13 / 7,1 / 2$ \\
\hline \multirow{4}{*}{ observer } & $\lambda_{1}, \lambda_{2}, \lambda_{3}$ & $20,600,600$ \\
\cline { 2 - 3 } & $\gamma_{1}, \gamma_{2}, \gamma_{3}$ & $40,800,800$ \\
\cline { 2 - 3 } & $m_{1}, m_{2}, m_{3}$ & $50,50,50$ \\
\cline { 2 - 3 } & $n_{1}, n_{2}, n_{3}$ & $0.001,0.01,20$ \\
\cline { 2 - 3 } & $\omega_{p}, \sigma_{4}$ & $10,1 / 2$ \\
\hline
\end{tabular}

The fuel cell electric vehicle behavior simulations are established using the parameters presented in Table 3. 
Table 3. Parameters about hybrid power vehicle

\begin{tabular}{|c|c|c|}
\hline Component & Parameter & Values \\
\hline \multirow{4}{*}{ Vehicle } & Total weight & $1625 \mathrm{Kg}$ \\
\cline { 2 - 3 } & Rolling resistance & 0.015 \\
\cline { 2 - 3 } & Drag coefficient & 0.26 \\
\cline { 2 - 3 } & Frontal area & $2.7 \mathrm{~m}^{\wedge} 2$ \\
\cline { 2 - 3 } & Rolling radius & 0.25 \\
\hline \multirow{2}{*}{$\begin{array}{c}\text { Electric } \\
\text { motor }\end{array}$} & Rated power & $75 \mathrm{KW}$ \\
\cline { 2 - 3 } Fuel \\
cell & Peak speed & $9000 \mathrm{r} / \mathrm{min}$ \\
\hline Battery & Maximum power & $50 \mathrm{KW}$ \\
\cline { 2 - 3 } & Rated voltage & $285 \mathrm{~V}$ \\
\hline Supercapacitor & Rated capacity & $30 \mathrm{Ah}$ \\
\hline DC-link bus & Rated voltage & $380 \mathrm{~V}$ \\
\hline \multirow{2}{*}{\begin{tabular}{c} 
Converter \\
\cline { 2 - 3 }
\end{tabular}} & Inductor $L_{b a t}, L_{f}$ & $0.0001 \mathrm{H}$ \\
\cline { 2 - 3 } & Capacitor $C_{b a t}$ & $0.0004 \mathrm{~F}$ \\
\hline
\end{tabular}

Urban Dynamometer Driving Schedule (UDDS), a standard driving cycle for vehicles homologation, is considered here. The simulation sampling period is set to $t_{\text {samp }}=60 \mathrm{us}$ which is the same as the actual switching frequency of the transistor. The LPM interval is defined as $[0 \mathrm{~W}, 850 \mathrm{~W}]$, the initial $K_{p}$ for SOC PI controller is 5000, and $K_{p}$ and $\mathrm{K}_{\mathrm{i}}$ for FC efficiency are 1000 and 0.01 , respectively. The output amplification factor of FLPT is 1000 for $K_{p}$.

First of all, in order to confirm the stability of the hybrid power systems of FCHEV, the tracking of real-time driving speed of the vehicle is shown in Figure 8(a). It shows that when the proposed method is used for energy management and control under driving conditions, the demand power is satisfied in real time which means that the vehicle can follow the speed of the reference driving cycle UDDS.

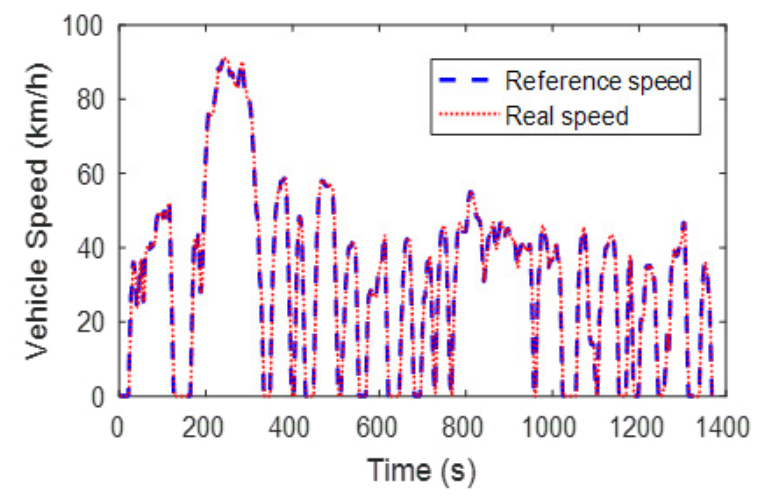

(a) The change of vehicle speed tracking UDDS

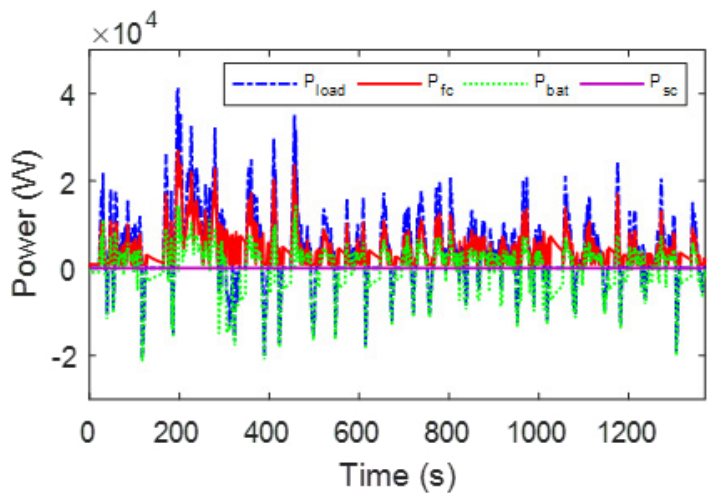

(b) The power flow curve of UDDS

Figure 8. The vehicle driving process diagram

By using EMS, the FC handles low frequencies positive power while also using the battery for fast and regenerative braking power transients which compensates for the slow dynamic characteristics of fuel cells and insufficient peak power. The output power of the SC has a small variation range because the bus voltage is controlled by battery, and the fast discharge characteristics of SC can assist the battery which is conducive to the protection of the battery.

Figure 9 and Table 4 display the vehicle hydrogen consumption using the proposed EMS for the UDDS driving cycle.

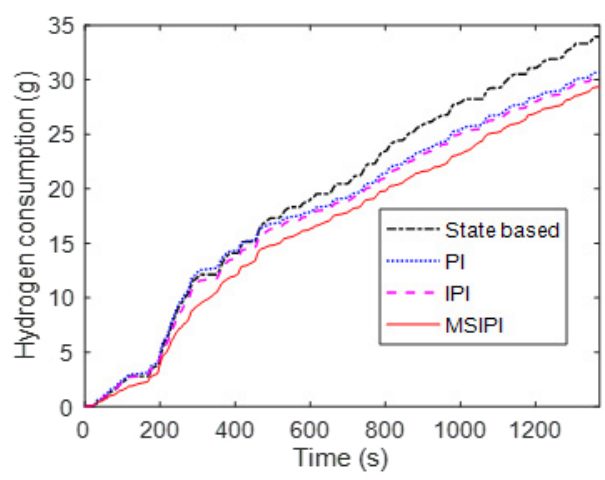

Figure 9. The $H 2$ consumption of different EMS

Table 4. The final $\mathrm{H} 2$ consumption of different EMS

\begin{tabular}{|c|c|}
\hline Method & Consumption $(\mathrm{g})$ \\
\hline State based & 33.9604 \\
\hline PI-EMS & 30.7000 \\
\hline IPI-EMS & 30.3196 \\
\hline MSIPI-EMS & 29.3816 \\
\hline
\end{tabular}

It can be noted that the developed multi-mode and improved PI (MSIPI) EMS has lower hydrogen consumption. As shown in Figure 10, this happens 
because its overall efficiency changes compactly, in comparison with the ones of the state-based, classical PI and improved PI EMS which change by $13.48 \%, 4.29 \%$ and $3.09 \%$, respectively.

Because using multiple modes and taking FLPT into consideration as an input to the PI controller, which maintains the fuel cell efficiency around $60 \%$, the proposed multi-mode EMS has a more compact fuel cell stark efficiency (see Figure 10). To compare the efficiency of fuel cell stacks under different EMS, the coefficient of variation which equals to the ratio of the standard deviation to the average is used to represent the changes in efficiency. As listed in Table 5, the coefficient of variance of the proposed multi-mode EMS is lower compared with the ones of the other three EMS. This means that the FC efficiency has less variability and is more concentrated when the load changes.
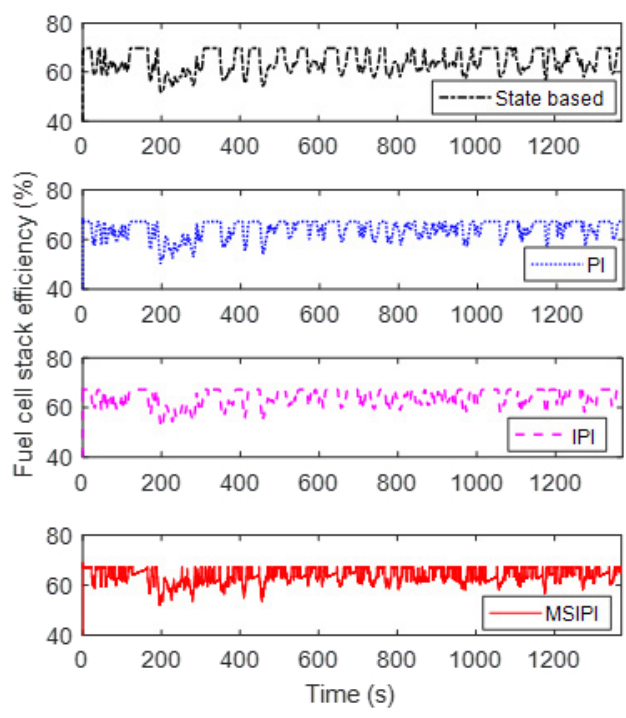

Figure 10. The power flow curve of UDDS

Table 5. Coefficient of variation of fuel cell stack efficiency

\begin{tabular}{|c|c|}
\hline Method & Coefficient of variation \\
\hline State based & 0.0710 \\
\hline PI-EMS & 0.0555 \\
\hline IPI-EMS & 0.0520 \\
\hline MSIPI-EMS & 0.0476 \\
\hline
\end{tabular}

As seen in Figure 11 and Table 6, the final SOC of multi-mode EMS can better converge to the initial SOC value $(60 \%)$. This also confirms the capability of the proposed method to impose SOC charge-sustainability. Meanwhile, the variation of the SOC of the battery ranges within the upper and lower limits, by using the proposed EMS. Compared with the PI and IPI EMS which lead to a very narrow utilization of the SOC of the battery, the multi-mode EMS deals with the proper utilization of the available energy of the battery subjected to its SOC constraints.

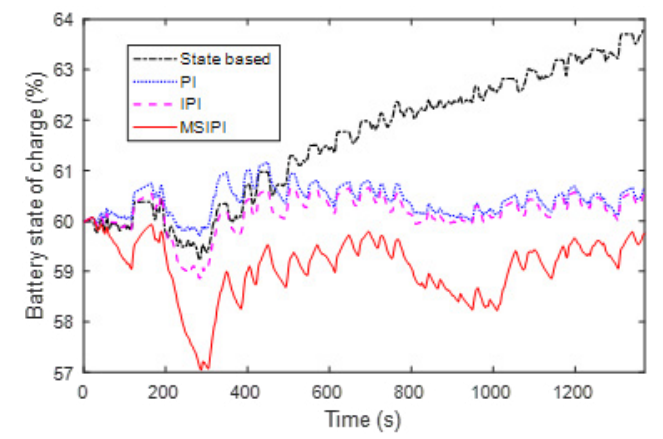

Figure 11. The battery state of charge under UDDS

Table 6. The final SOC of battery

\begin{tabular}{|c|c|}
\hline Method & End SOC (\%) \\
\hline State based & 63.7952 \\
\hline PI-EMS & 60.6345 \\
\hline IPI-EMS & 60.5220 \\
\hline MSIPI-EMS & 59.7622 \\
\hline
\end{tabular}

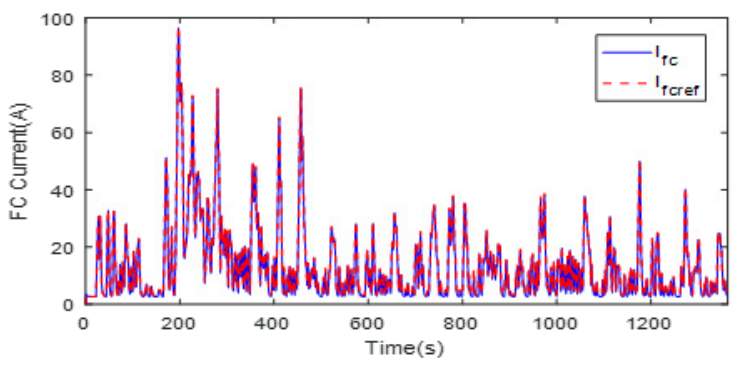

Figure 12. The tracking effect on FC

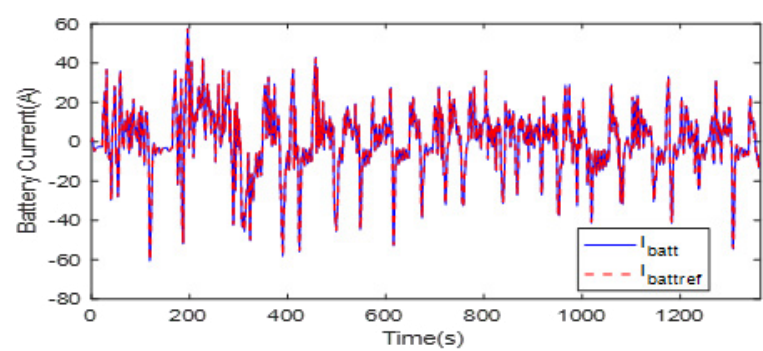

Figure 13. The tracking effect on battery

For the current variable controls under the UDDS driving cycle, the FC current and battery current are well controlled because they track their reference values delivered by the proposed EMS, and the DC bus voltage loop. This is shown in Figures 12 and 13 which display the good performances of the designed controller. 
This also guarantee the stability of whole loop. In the tracking error view, it can be seen that the tracking error of the FC current and battery current are smaller than 0.2 and 0.5 which can be seen from Figure 14.
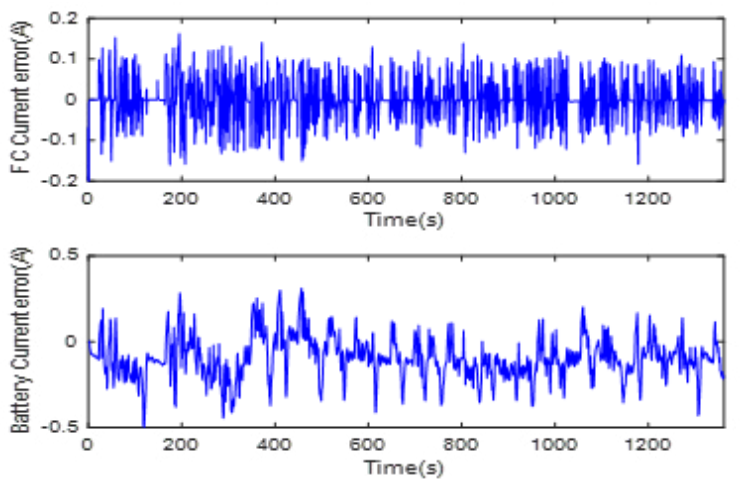

Figure 14. The tracking error of power source current

The time changes in DC bus voltage are employed to verify the performance of the proposed modelfree controller, as shown in Figure 15 which compares the PI and MFCFTSMC. It can be concluded that the PI controller shows higher voltage oscillations, particularly when the power flow changes drastically. In contrast, the proposed MFCFTSMC is better at maintaining bus voltages under severe load changes which is also more stable than PI controller. That means that the battery can help fuel cells meet the demand power and improve the efficiency of the drive motor through the proposed controller. Also, from Figure 15, one can see that the DC bus voltage is stable around $380 \mathrm{~V}$ even under the time-varying environment.

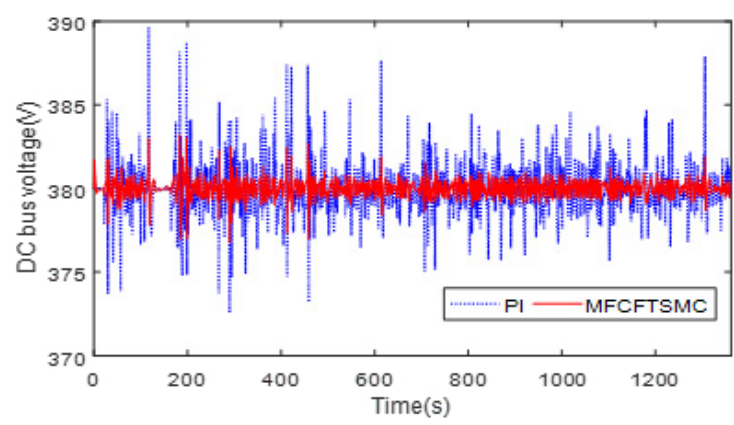

Figure 15. DC Bus voltage tracking comparison

Figure 16(a) demonstrates the results of sliding mode observer of HOFTSM in fuel cell hybrid power system. Figure 16(b) shows the observation error results of state variable. It is known that the estimated error of power sources current and DC bus voltage is small.
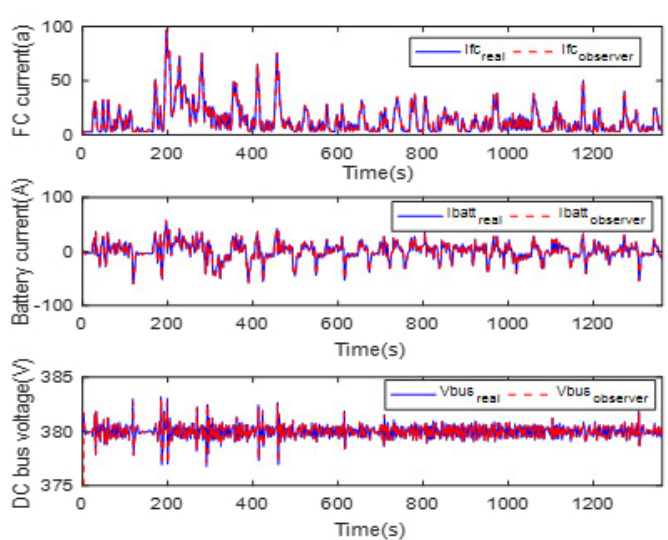

(a) State variable observer performance

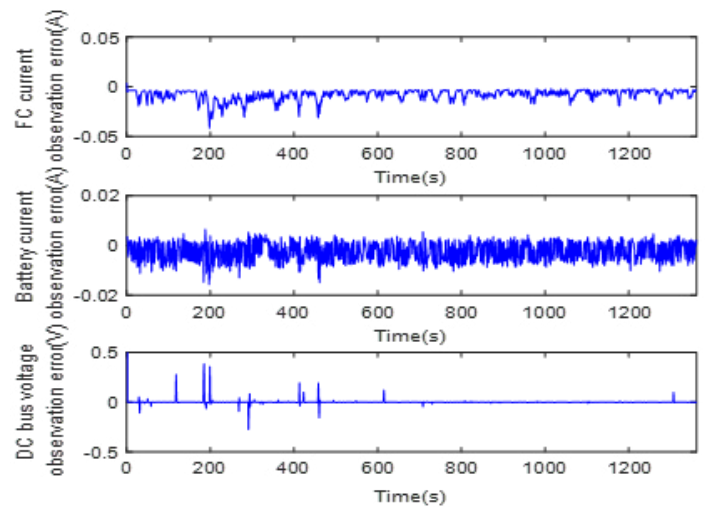

(b) Observation error

Figure 16. The observation of state variable

\section{Conclusion}

This paper focuses on the control and energy management of FCHEV. Firstly, in order to reduce the dependence on the model information, based on the model-free control methods, the ultra-local model of fuel cell hybrid system is constructed based on the input and output of the system which take into account its nonlinearities.

Secondly, it proposes the control of two power converters (FC and Battery). The model-free continuous fast terminal sliding mode control method is proposed and adopted to design the DC bus voltage controller and sources current controller, and the high-order fast terminal sliding mode observer is designed to estimate the unknown terms of the ultra-local model. The simulation results prove that the designed model free controller with sliding mode observer has the advantages of better robustness and good tracking performance in the case of load fluctuations, advantages which meet the control objectives. 
Finally, the energy management strategy (EMS) that allows an appropriate distribution of the power demanded by the load between the three sources is considered. A multi-mode EMS has been developed according to different objectives and application scenarios. Compared with other three strategies, the designed strategy has the lowest consumption and a better stability of fuel cell efficiency.

In future studies, the rule-based EMS combined with real-time optimization method will be studied and developed. Meanwhile, as regards the control

\section{REFERENCES}

Aouzellag, H., Ghedamsi, K., Aouzellag, D. (2015). Energy management and fault tolerant control strategies for fuel cell/ultra-capacitor hybrid electric vehicles to enhance autonomy, efficiency and life time of the fuel cell system, International Journal of Hydrogen Energy, 40(22), 7204-7213.

Armghan, H., Ahmad, I., Ali, N., Munir, M. F., Khan, S. \& Armghan, A. (2018). Nonlinear Controller Analysis of Fuel Cell-Battery-Ultracapacitor-based Hybrid Energy Storage Systems in Electric Vehicles, Arabian Journal for Science and Engineering, 43, 3123-3133.

Barkat, A., Marinescu, B., Join, C. \& Fliess, M. (2018). Model-free control for VSC-based HVDC systems. In 2018 IEEE PES Innovative Smart Grid Technologies Conference Europe (ISGT-Europe), (pp. 1-6).

Bendjedia, A. M., Phillips, A. B., Turnock, S. R. \& Wilson, P. A. (2017). Development of a multi-scheme energy management strategy for a hybrid fuel cell driven passenger ship, International Journal of Hydrogen Energy, 42, 623-635.

Benmouna, A., Becherif, M., Depernet, D., Depature, C. \& Boulon, L. (2020). Nonlinear control and optimization of hybrid electrical vehicle under sources limitation constraints, International Journal of Hydrogen Energy, 45(19), 11255-11266.

Das, H. S., Tan, C. W. \& Yatim, A. H. M. (2017). Fuel cell hybrid electric vehicles: A review on power conditioning units and topologies, Renewable and Sustainable Energy Reviews, 76, 268-291.

Depature, C., Lhomme, W., Sicard, P., Bouscayrol, A. \& Boulon, L. (2017). Real-Time Backstepping Control for Fuel Cell Vehicle Using Supercapacitors, IEEE Transactions on Vehicular Technology, 67(1), 306-314.

Fadil, H. E., Giri, F., Guerrero, J. M. \& Tahri, A. (2014). Modeling and Nonlinear Control of a Fuel of electric motor, the high-performance robust controller will be examined.

\section{Acknowledgments}

This research proposed in this paper has been partially supported by the Intergovernmental International Science and Technology Innovation Cooperation Key Project of National Key R\&D Program (grant 2021YFE0102700) and by the Natural Science Foundation of Jiangsu Province (grant BK20170094).

Cell/Supercapacitor Hybrid Energy Storage System for Electric Vehicles, IEEE Transactions on Vehicular Technology, 63(7), 3011-3018.

Fliess, M. \& Join, C. (2013). Model-free control, International Journal of Control, 86(12), 2228-2252.

Hilairet, M., Ghanes, M., Béthoux, O., Tanasa, V., Barbot, J. \& Normand-Cyrot, D. (2013). A passivitybased controller for coordination of converters in a fuel cell system, Control Engineering Practice, 21(8), 1097-1109.

Larminie, J., Dicks, A. \& McDonald, M S. (2003). Fuel Cell Systems Explained. Chichester, UK. John Wiley.

Mane, S., Mejari, M., Kazi, F. \& Singh, N. M. (2017). Improving Lifetime of Fuel Cell in Hybrid Energy Management System by Lure-Lyapunov-Based Control Formulation, IEEE Transactions on Industrial Electronics, 64(8), 6671-6679.

Marzougui, H., Amari, M., Kadri, A., Bacha, F. \& Ghouili, J. (2017). Energy management of fuel cell/ battery/ultracapacitor in electrical hybrid vehicle, International Journal of Hydrogen Energy, 42(13), 8857-8869.

Mustafa, G. I. Y., Wang, H. P. \& Tian, Y. (2019). Model-free adaptive fuzzy logic control for a half-car active suspension system, Studies in Informatics and Control, 28(1), 13-24. DOI: 10.24846/v28i1y201902

Pany, P., Singh, R. K. \& Tripathi, R K. (2016). Active load current sharing in fuel cell and battery fed DC motor drive for electric vehicle application, Energy Conversion \& Management, 122, 195-206.

Song, K., Li, F., Hu, X., He, L., Niu, W., Sihao, L. \& Zhang, T. (2018). Multi-mode energy management strategy for fuel cell electric vehicles based on driving pattern identification using learning vector quantization neural network algorithm, Journal of Power Sources, 389, 230-239. 
Tahri, A., Fadil, H. E., Belhaj, F. Z., Gaouzi, K., Rachid, A., Giri, F. \& Chaoui, F. Z. (2018). Management of fuel cell power and supercapacitor state-of-charge for electric vehicles, Electric Power Systems Research, 160, 89-98.

Twidell, J. \& Weir, T. (2015) Renewable Energy Resources. London. Routledge.

Venkatesh, V. \& Kamalakannan, C. (2016). Real Time Analysis of Positive Output Super Lift Converter Using ANN Controller, Studies in Informatics and Control, 25(3), 323-334. DOI: 10.24846/v25i3y201606

Venkatesh, V. \& Kamalakannan, C. (2020). Analysis and Implementation of Positive Output Super Lift Converter Using Artificial Neural Network with Sliding Mode Controller, Studies in Informatics and Control, 29(3), 373-383. DOI: 10.24846/ v29i3y202010

Vural, B., Dusmez, S., Uzunoglu, M., Ugur, E. \& Akin, B. (2014). Fuel Consumption Comparison of Different Battery/Ultracapacitor Hybridization Topologies for Fuel-Cell Vehicles on a Test Bench, IEEE Journal of Emerging and Selected Topics in Power Electronics, 2(3), 552-561.

Wang, B., Luo, C., Yu, Y., Wang, G. \& Xu, D. (2018). Antidisturbance Speed Control for Induction Machine Drives Using High-Order Fast Terminal Sliding-Mode Load Torque Observer, IEEE Transactions on Power Electronics, 33(9), 7927-7937.
Wilberforce, T., El-Hassan, Z., Khatib, F., Makky, A. A., Baroutaji, A., Carton, J. G. \& Olabi, A. G. (2017). Developments of electric cars and fuel cell hydrogen electric cars, International Journal of Hydrogen Energy, 42(40), 25695-25734.

Xu, D., Liu, Q., Yan, W. \& Yang, W. (2019a). Adaptive Terminal Sliding Mode Control for Hybrid Energy Storage Systems of Fuel Cell, Battery and Supercapacitor, IEEE Access, 7, 29295-29303.

Xu, L., Ouyang, M., Li, J, Yang, F., Lu, L. \& Hua, J. (2013). Optimal sizing of plug-in fuel cell electric vehicles using models of vehicle performance and system cost, Applied Energy, 103, 477-487.

Xu, W., Junejo, A. K., Liu, Y. \& Islam, M. R. (2019b). Improved Continuous Fast Terminal Sliding Mode Control with Extended State Observer for Speed Regulation of PMSM Drive System, IEEE Transactions on Vehicular Technology, 68(11), 10465-10476.

Zhao, K., Yin, T., Zhang, C., He, J., Li, X., Chen, Y., Zhou, R. \& Leng, A. (2019) Robust Model-Free Nonsingular Terminal Sliding Mode Control for PMSM Demagnetization Fault, IEEE Access, 7, 15737-15748. 